\title{
PROCEEDINGS REPORTS
}

\section{Challenges and rewards of the clinician scientist career path}

\author{
Greg MT Hare MD, PhD
}

\author{
Department of Anesthesia, \\ University of Toronto \\ Presented at the CSCI Symposium \\ Clinician Investigators: Pathway to Future Success \\ Winnipeg, September 2007
}

Clin Invest Med 2008; 31 (5): E302-E303.
The pathway to a successful career as a Clinician Scientist has no shortage of challenges. However, most of those who embark upon this difficult journey are rewarded for their efforts. The following summarizes one perspective on how to manage the challenges and enjoy the rewards of a career as a Clinician Scientist.

The first suggestion is to obtain the best training possible with the most appropriate and accomplished supervisor in an area of particular interest to the trainee. Most $\mathrm{MD} / \mathrm{PhD}$ candidates have achieved this early in their program. Pick a question of importance that will hold your interest and become your passion. Unfortunately, the road is long, with many difficulties and few short-cuts. You must obtain excellence in two often very different realms: Clinical medicine and basic research. To compete and perform optimally in both realms you must be exceptionally trained.

The second suggestion is to find clinical and scientific mentors who will support you throughout your career. The mentor-student relationship must benefit the student and ideally will last the length of your career. If an excellent mentor is not excellent for the student, then the student must seek additional mentors. As you progress in your training you may benefit by interacting with a number of different mentors.
The third suggestion is that you seek an academic appointment in a Department that will support your efforts and provide adequate resources and protected time. The Chair, and other Department members must support your need for time and money. Time is of utmost importance. Without time, you will not be able to build a laboratory, produce new research and achieve peer reviewed funding. This can be a slow process and you may need years of support before your begin to see your productivity and achievements grow.

The fourth suggestion is to learn how to deal with many of the difficulties associated with academic medicine including, lack of rapid progress in your academic program, unsuccessful grants and rejected manuscript submissions. Many good ideas may not gain immediate acceptance. Successful granting often requires that you propose a novel idea, but not too novel an idea. With increasing competition for existing peer reviewed funding, each successful grant may be preceded by 4 or 5 unsuccessful grants. The bar for success is always moving up. Persistence is necessary and is usually rewarded. It is encouraging to hear many senior investigators relate that one of their grants that was poorly reviewed one year became the top grant on a subsequent submission. 
Finally, the measure of success must be set by the candidate. The only sure reward is that you are embarking on a career which will enable you to achieve academic goals of your choosing. Your career choice is a marathon, not a sprint. Remember to keep your focus on what you have accomplished. Make your next short term goal realistic and slowly progress towards more challenging long-term goals. Do not be discouraged by the fact that others may have achieved much more than you. Focus on your goals and enjoy your accomplishments and successes.

Correspondence to:

Gregory MT Hare MD, PhD.

Department of Anesthesia, University of Toronto,

St. Michael's Hospital,

Shuter Wing,

$1028 \mathrm{f} 30$ Bond Street,

Toronto, ON

M5B 1W8

hareg@smh.toronto.on.ca 